\title{
Potencial inseticida do extrato etanólico de Anadenanthera macrocarpa (BENTH.) em lepidópteros-praga
}

\author{
Glauciany Soares LOPES ${ }^{1}$, Luciana Barboza SILVA ${ }^{1}$, Eliane CARNEIRO ${ }^{1}$, \\ Manoel Lopes da SILVA FILHO ${ }^{1}$, João Sammy Nery de SOUZA ${ }^{1}$, Fernandes Antonio ALMEIDA ${ }^{2}$, \\ Bruno Ettore Pavan ${ }^{3}$
}

\author{
${ }^{1}$ Universidade Federal do Piauí, Bom Jesus, PI, Brasil. \\ ${ }^{2}$ Universidade Federal de Campina Grande, Pombal, PB, Brasil. \\ ${ }^{3}$ Faculdade de Engenharia, Universidade Estadual Paulista, Ilha Solteira, SP, Brasil \\ *E-mail: elian.cbs@hotmail.com
}

Recebido em janeiro/2019; Aceito em maio/2019.

\begin{abstract}
RESUMO: O complexo de lagartas desfolhadoras - Spodoptera cosmioides, Spodoptera frugiperda e Helicoverpa armigera, são responsáveis por uma considerável redução na produção de grãos. A aplicação de extratos botânicos consiste em uma alternativa viável e eficiente de controle, além de ser menos nociva ao meio ambiente e a saúde do homem. O objetivo foi avaliar o potencial inseticida do extrato bruto de folhas e casca de Anadenanthera macrocarpa, sobre as lagartas de S. frugiperda, S. cosmioides, H. armigera. Os insetos foram mantidos em dieta artificial em condições controladas. Foi avaliada a ação toxica dos extratos por aplicação tópica. Primeiramente foi estimada a concentração letal e em seguida foram determinados os parâmetros biológicos das três espécies de lepidópteros. Os extratos etanólicos de folhas e casca de $A$. macrocarpa foram eficientes no controle de H. armigera, S. frugiperda e S. cosmioides reduzindo a sobrevivência em 75, 60 e 50\%, respectivamente, além de alterações nos parâmetros biológicos como, aumento do período larval, redução de peso das larvas e pupas. Os compostos químicos presentes nos extratos etanólicos de folhas e casca de $A$. macrocarpa, além de causar mortalidade direta dos insetos estudados, alteram o ciclo biológico de S. frugiperda, S. cosmoides e H. armigera.
\end{abstract}

Palavras-chave: Angico-preto; Spodoptera cosmioides; Spodoptera frugiperda; Helicoverpa armigera; bioinseticidas.

\section{Potential insecticide of the ethanolic extract of Anadenanthera macrocarpa (BENTH.) in lepidopteran-pests}

\begin{abstract}
The leafless worm complex - Spodoptera cosmioides, Spodoptera frugiperda and Helicoverpa armigera - are responsible for a considerable reduction in grain production. The application of botanical extracts is a viable and efficient alternative of control, besides being less harmful to the environment and human health. The objective was to evaluate the insecticidal potential of the raw extract of leaves and bark of Anadenanthera macrocarpa, on S. frugiperda, S. cosmioides, H. armigera. The insects were kept on artificial diet under controlled conditions. The toxic action of the extracts was evaluated by topical application. First the lethal concentration was estimated and then the biological parameters of the three lepidopteran species were determined. The extracts of leaves and bark of A. macrocarpa were efficient in the control of H. armigera, S. frugiperda and S.a cosmioides, reducing survival in 75,60 and $50 \%$, respectively, as well as changes in biological parameters such as larval period increase, weight reduction of larvae and pupae. The chemical compounds present in the leaves and bark extracts of A. macrocarpa, besides causing direct mortality of the studied insects, alter the biological cycle of S. frugiperda, S. cosmoides and H. armigera.
\end{abstract}

Keywords: Angico-black; Spodoptera cosmioides; Spodoptera frugiperda; Helicoverpa armígera; bioinsecticides.

\section{INTRODUÇÃO}

O cultivo de grãos sofre danos constantes causados por insetos desfolhadores, como Spodoptera cosmioides Walker), Spodoptera frugiperda (Smith) e Helicoverpa armigera (Hübner) (Lepidoptera: Noctuidae) (CABEZAS et al., 2013; CZEPAK et al., 2013). Lepidópteros-praga polífagos, tais como $S$. cosmioides e $S$. frugiperda são pragas chave de culturas importantes como soja e milho (BUENO et al., 2011; DEQUECH et al., 2013). Helicoverpa armigera ataca as folhas, flores e vagens das plantas de soja, algodão, tomate, feijão (SPECHT et al., 2013; GUAZINA et al., 2019).

Uma estratégia de manejo mal definida conciliada com uso abusivo de inseticidas (BUENO et al., 2011), podem acarretar surtos populacionais dos insetos além de eliminar os inimigos naturais (HARDSTONE; SCOTT, 2010). Estes problemas vêm estimulando pesquisas com compostos alternativos visando produção agrícola mais sustentável e eficiente (REGNAULT-ROGER et al., 2012). 
Dentro desta perspectiva, os extratos botânicos podem ser uma alternativa de controle, pois são uma fonte rica de moléculas bioativas (ISMAN, 2006; SANTOS et al., 2015), com potencial tóxico a pragas e doenças (FOUAD et al., 2014; MELO et al., 2015; CARVALHO NETO et al., 2017; ALMEIDA et al., 2016).

As plantas da família Fabaceae, Malvaceae e Meliaceae são consideradas as principais fontes de substâncias bioinseticidas devido à grande diversidade de compostos secundários (aleloquímicos) sintetizados e acumulados em diferentes partes da planta (VIEGAS-JÚNIOR, 2003; DINESH et al., 2014).

O extrato obtido a partir do angico - preto [Anadenanthera macrocarpa (Benth.) Brenan], apresenta toxicidade sobre lagartas desfolhadoras (CARVALHINHO et al., 2017) e Callosobruchus maculatus F. (Coleoptera: Chrysomelidae: Bruchinae) (MELO et al., 2015). O Extrato da casca de [Anadenanthera pavonina L.] (Fabaceae) também apresenta efeito letal em Diatraea saccharalis (Fabricius) (Lepidoptera: Pyralidae), esta é a principal praga da cana-de-açúcar, causando danos aos colmos. Esta espécie praga é encontrada no Caribe, na América Central, no hemisfério ocidental e nas regiões mais quentes da América do Sul (SILVA et al., 2012).

O objetivo deste estudo foi avaliar o potencial bioinseticida do extrato bruto de folhas e casca de angico-preto ( $A$. macrocarpa), sobre as lagartas de H. armigera, $S$. frugiperda, S. cosmioides.

\section{MATERIAL E MÉTODOS}

2.1. Criação de insetos

A criação foi estabelecida, a partir de coletas em campos de produção de soja e milho durante a safra 2014/2015 na região do alto médio Gurgueia Bom Jesus - PI, Brasil. Foram coletadas três espécies de lagartas desfolhadoras devidamente identificadas pelo pesquisador Dr. Sergio Ide, do Instituto Biológico, SP.: H. armigera, S. cosmioides e S. frugiperda. As espécies foram criadas no Laboratório de Fitotecnia (CPCE/UFPI), em condições controladas $\left(25 \pm 5^{\circ} \mathrm{C}, 60 \pm 10 \%\right.$ $\mathrm{UR}, 12: 12 \mathrm{LD})$. Os adultos foram acondicionados em gaiolas de PVC $(30$ x $20 \mathrm{~cm} \varnothing)$ e alimentados com solução de mel a $10 \%$. A cada dois dias foram coletadas as posturas e acondicionadas em copos plásticos até a eclosão das lagartas das três espécies estudadas, as quais foram transferidas para a dieta artificial adaptada de Kasten Jr. et al. (1978).

\subsection{Espécies Vegetais e Preparação do extrato bruto}

Os extratos foram preparados no Laboratório de Química Orgânica da Universidade Federal do Piauí, Campus Prof ${ }^{a}$ Cinobelina Elvas, Bom Jesus-PI, a partir da casca e folhas de angico-preto coletadas na cidade de Angical (PI) $\left(06^{\circ} 05^{\prime} 09^{\prime}\right.$ 'S, $42^{\circ} 44^{\prime} 20^{\prime \prime}$ ), nos meses de agosto e outubro de 2014. O material vegetal foi identificado no "Herbário Graziela Barroso" localizado na Universidade Federal do Piauí, Campus Ministro Petrônio Portela, Teresina-PI, exsicata $n^{\circ}$ 21.643TEPB.

Para o preparo dos extratos, as cascas e folhas passaram pelo processo de limpeza para retirada de impurezas, folhas danificadas ou com sintomas visuais de doença, insetos presentes e outros organismos. Logo após, foi realizada a présecagem do material vegetal durante três dias em estufa de circulação forçada a $60{ }^{\circ} \mathrm{C}$. O material seco foi triturado em moinho de faca para a obtenção de um pó e levado, novamente, à estufa de circulação para uma nova secagem, a $60{ }^{\circ} \mathrm{C}$ por 72 h. O material foi, então, pesado em balança analítica de precisão, sendo utilizados $1230 \mathrm{~g}$ de folhas e galhos finos e $450 \mathrm{~g}$ de casca, os quais foram embebidos em etanol (1: $5 \mathrm{p} / \mathrm{v})$ num balão hermeticamente fechado durante três dias. As amostras foram então filtradas através de papel de filtro, procedimento repetido por 3 vezes. O etanol da solução filtrada foi removido em um evaporador rotativo a $50^{\circ} \mathrm{C}$ a vácuo de $-600 \mathrm{~mm} \mathrm{Hg}$. Após a evaporação do solvente em uma câmara aerada, o rendimento foi determinado pesando-se os extratos de cada parte da planta de A. macrocarpa, isto é 70,71 $\mathrm{g}$ do extrato etanólico das folhas e 30,47 g do extrato etanólico da casca.

\subsection{Bioensaios}

Os ensaios experimentais foram desenvolvidos no laboratório de Fitotecnia do Campus Professora Cinobelina Elvas da Universidade Federal do Piauí, no período de agosto de 2014 a junho de 2015 na cidade de Bom Jesus, Piauí, em condições controladas (temperatura $25 \pm 2{ }^{\circ} \mathrm{C}, 70 \pm 10 \% \mathrm{UR}$, 12:12 h (L:D), com delineamento inteiramente casualizado, cinco repetições contendo 20 insetos cada, além da testemunha que foi exposta apenas ao solvente etanol.

Para obtenção da solução estoque com concentração 250 $\mathrm{mg} \mathrm{L}^{-1}$ dos extratos brutos, pesou-se, em balança de precisão a massa do produto em proveta de $10 \mathrm{~mL}$ e o volume final foi completado com solvente para $10 \mathrm{~mL}$ (etanol foi utilizado como solvente). A partir desta solução foram preparadas diluições seriadas, obtendo-se, assim, diferentes concentrações.

Testes preliminares foram realizados para se determinar a faixa de resposta, ou seja, o intervalo de concentrações dos extratos que causa mortalidades superiores a zero, mas inferiores a $100 \%$, equivalente a $0,250,500$ e $1000 \mathrm{mg}^{-1}$.

Com auxílio de pipeta graduada, foram aplicados $10 \mu \mathrm{L}$ de solução dos extratos diluídos em etanol, no mesotórax entre o segundo e o terceiro par de pernas em lagartas de terceiro ínstar de $S$. cosmioides, S. frugiperda e $H$. armigera, para cada concentração, em três repetições. O tamanho de gota escolhido foi para assegurar uma cobertura eficiente, sem que ocorresse perda do produto por escorrimento. Vinte insetos foram então expostos por um período de 48 horas, em potes plásticos de $100 \mathrm{~mL}$ ( $5 \mathrm{~cm}$ de diâmetro X $6 \mathrm{~cm}$ de altura) contendo dieta artificial, sendo mantidas em condições de laboratório (25 \pm 2 ${ }^{\circ} \mathrm{C}, 70 \pm 10 \% \mathrm{UR}, 12: 12 \mathrm{~h}$ ). Após este período avaliou-se a mortalidade proporcionada pelas concentrações dos respectivos extratos vegetais utilizados, além da testemunha.

Após a realização dos testes preliminares, procedeu-se à realização do bioensaio para a detecção da toxicidade, que consistiu de 20 lagartas com 5 repetições, por tratamento (concentração). Foi utilizada a $\mathrm{CL}_{50}$ (concentração letal para $50 \%$ dos indivíduos) aproximada dos extratos com base na faixa de resposta dos testes preliminares. Seguindo o mesmo método acima descrito, $10 \mu \mathrm{L}$ de solução dos extratos diluídos em etanol, no mesotórax entre o segundo e o terceiro par de pernas em lagartas de terceiro instar de $S$. cosmioides, $S$. frugiperda e H. armigera. A testemunha foi constituída apenas com o solvente (etanol). Após a aplicação, as lagartas de terceiro ínstar, foram transferidos para potes plásticos de 100 $\mathrm{mL}$ contendo dieta artificial, sendo mantidas em condições de laboratório.

Avaliaram se a sobrevivência larval, peso larval, duração da fase larval, a duração, peso e sobrevivência das pupas formadas e viabilidade dos adultos. A sobrevivência larval foi 
avaliada a cada 24 horas e o peso larval após 10 dias do início da aplicação dos extratos até o $16^{\circ}$ dia a cada 48 horas.

\subsection{Análise estatística}

Os resultados de mortalidade foram submetidos à análise de Probit (FINNEY, 1971), por intermédio do procedimento PROC PROBIT do programa System of Statistical Analyses (SAS) (SAS INSTITUTE, 2002), gerando-se assim as curvas de concentração-mortalidade. Os dados de mortalidade obtidos foram corrigidos pela mortalidade que ocorreu na testemunha. Efetuou-se análise de regressão para a $\mathrm{CL}_{50} \mathrm{e}$ análise de variância dentro de cada espécie de inseto, para as variáveis peso e duração, comparando as médias pelo teste de Tukey a $5 \%$ de probabilidade. As análises foram efetuadas utilizando o programa System of Statistical Analyses (SAS) (SAS INSTITUTE, 2002).

\section{RESULTADOS}

Os extratos etanólicos das folhas e casca de $A$. macrocarpa nos bioensaios de aplicação tópica causaram mortalidade e alterações nos parâmetros biológicos avaliados (longevidade larval, massa das larvas e pupas) sobre as lagartas $S$. frugiperda, S. cosmioides e H. armigera.

O extrato etanólico da folha de A. macrocarpa foi 2,4 e 1,5 vezes mais tóxico do que o extrato da casca para $S$. frugiperda e $S$. cosmioides na $\mathrm{CL}_{50}$, respectivamente (Tabela 1 ). O extrato da casca e folha apresentaram toxicidade similar a $H$. armigera. Comparado os valores de $\mathrm{CL}_{50}$ dos extratos entre as espécies, constata-se que o extrato etanólico da folha foi mais tóoxico a $S$. frugiperda, e o extrato da casca a $H$. armigera (Tabela 1).
O tempo letal mediano $\left(\mathrm{TL}_{50}\right)$ do extrato etanólico não apresentou diferença entre extratos de folhas e casca de $A$. macrocarpa (Tabela 2). Sendo que ambos os extratos apresentaram redução de tempo para todas as espécies estudadas quando comparada a testemunha (Tabela 2). Observando o tempo de ação do extrato sobre o inseto, podese inferir que os dois extratos de A. macrocarpa têm o mesmo potencial toxicológico, embora com ação diferente entre as espécies.

A partir da análise de sobrevivência, construída por meio do estimador, foi possível constatar que os extratos etanólicos causaram redução da sobrevivência dos lepidópteros aqui utilizados como espécie alvo. Os extratos provenientes da casca e das folhas apresentaram resultados semelhantes, porém, o comportamento das espécies foi diferente em resposta aos extratos, apresentando redução da sobrevivência para $H$. armigera, S. frugiperda e $S$. cosmioides de aproximadamente 75,60 e 50\%, respectivamente, quando comparados com o tratamento controle (Figura 1).

Apenas o extrato da folha de A. macrocarpa apresentou efeito sobre a longevidade larval para $S$. frugiperda, as outras espécies não foram afetadas. Também não houve efeito significativo dos extratos sobre a longevidade das pupas para as espécies estudadas (Tabela 3 ).

Os tratamentos de folhas e das cascas de A. macrocarpa apresentaram efeito significativo na redução da massa larval e de pupas em $S$. frugiperda e $S$. cosmioides, enquanto para $H$. armigera apenas os extratos da folha apresentaram redução significativa (Tabela 4).

Tabela 1. Concentração Letal do extrato etanólico das folhas e casca de Anadenanthera macrocarpa (Fabaceae: Mimosoideae) para Spodoptera frugiperda, Spodoptera cosmioides e Helicoverpa armigera.

Table 1. Lethal concentration of the ethanolic extract of the leaves and bark of Anadenanthera macrocarpa (Fabaceae: Mimosoideae) for Spodoptera frugiperda, Spodoptera cosmioides and Helicoverpa armigera.

\begin{tabular}{cccccc}
\hline \multirow{2}{*}{ Tratamento } & \multicolumn{2}{c}{ Concentração Letal } & \multirow{2}{*}{ Inclinação \pm DP } & \multirow{2}{*}{$\mathrm{X}^{2}$} & \multirow{2}{*}{$\mathrm{P}$} \\
\cline { 2 - 3 } & CL50 (IC 95\%) & CL95 (IC 95\%) & Spodoptera frugiperda & \\
\hline Folha & $3,72(3-4,7)$ & $24,66(17-43)$ & $2,00 \pm 0,19$ & 6,67 & $0,15^{* *}$ \\
Casca & $9,07(7,2-11)$ & $31,07(21-70)$ & $3,08 \pm 0,59$ & 0,32 & $0,98^{* *}$ \\
\hline \multicolumn{5}{c}{ Spodoptera Cosmioides } \\
\hline Folha & 91,72 & $2,08 \pm 0,17$ & 9,21 & $0,10^{* *}$ \\
Casca & $14,83(12-17)$ & 50,19 & $4,73 \pm 0,54$ & 8,75 & $0,27^{* *}$ \\
\hline Folha & $22,55(20-25)$ & Helicoverpa armigera & 8,38 & $0,29^{* *}$ \\
Casca & $6,42(5,6-73)$ & $31,39(25-40,7)$ & $2,39 \pm 0,16$ & 8,38 & $0,29^{* *}$ \\
\hline
\end{tabular}

$\mathrm{DP}=$ desvio padrão; $\mathrm{X}^{2}=$ qui-quadrado; $\mathrm{IC}=$ intervalo de confiança; $\mathrm{P}=$ probabilidade, $\mathrm{CL}_{50}=$ Concentração letal a $50 \%$ da população e $\mathrm{CL}$ $95=\mathrm{Concentração} \mathrm{letal}$ a $95 \%$ da população. ${ }^{*}$ Significativo a $1 \%$ de probabilidade.

Tabela 2. Tempo letal ( \pm EPM) para Spodoptera frugiperda, Spodoptera cosmioides e Helicoverpa armigera, após a aplicação tópica do extrato etanólico das folhas e casca de Anadenanthera macrocarpa (Fabaceae: Mimosoideae).

Table 2. Lethal time ( \pm SEM) for Spodoptera frugiperda, Spodoptera cosmioides and Helicoverpa armigera, after topical application of the leaves and bark extract of Anadenanthera macrocarpa (Fabaceae: Mimosoideae).

\begin{tabular}{cccc}
\hline & \multicolumn{3}{c}{ Tempo Letal Mediano (dias) } \\
\cline { 2 - 4 } & Controle & Folha & Casca \\
\hline H. armigera & $28,83 \pm 0,59$ & $9,03 \pm 0,51$ & $11,33 \pm 0,62$ \\
S. cosmioides & $30,17 \pm 0,62$ & $18,50 \pm 0,81$ & $21,55 \pm 0,74$ \\
S. frugiperda & $32,16 \pm 0,46$ & $20,64 \pm 0,76$ & $19,23 \pm 0,78$ \\
\hline
\end{tabular}

\section{DISCUSSÃO}

A atividade bioinseticida apresentada por Anadenanthera macrocarpa para os insetos avaliados é comum em plantas da família Fabaceae por apresentarem uma diversidade de compostos secundários como terpenoides, flavonoides, alcaloides e taninos que são sintetizados e acumulados em diferentes partes da planta (CHAN et al., 2013; DINESH et al., 2014; SARTORI et al., 2014).

Estes compostos naturais apresentam papel fundamental na defesa das plantas, possuem ação antimicrobiana produzidos em resposta ao ataque de microrganismos (FONSECA et al., 2017) e compostos anti-herbivoria que impedem ataques de herbívoros (WAR et al., 2011). 
Os resultados da atividade inseticida dos extratos utilizados sobre os lepidópteros testados durante a fase larval foram evidenciados por Matos et al. (2006), onde utilizaram extratos orgânicos de Trichilia spp. (Meliaceae) em $S$. frugiperda obtiveram mortalidade larval superior a $60 \%$. A atividade bioinseticida que resulta na alta mortalidade na fase larval é um resultado positivo, pois, estes efeitos nos estágios iniciais reduzem os prejuízos agrícolas diretos e ainda impedem a disseminação destas espécies-praga.

A atividade de extratos sobre a longevidade larval também foi relatada por Santos et al. (2008) onde observaram a redução da sobrevivência, duração e peso das fases larval e pupal de $S$. frugiperda exposta a extrato de Ricinus communis $L$. a inibição do crescimento pode ser devido à redução da ingestão de alimentos e baixa conversão de nutrientes para o crescimento, assim o alongamento da duração do período larval é um mecanismo que pode compensar a redução da ingestão de alimento em função de um ou mais inibidores presentes na dieta (MARTINEZ; ENDEM, 2001). Portanto, o prolongamento do período larval pode estar relacionado a mecanismos de compensação do inseto para assimilar a quantidade de nutrientes necessária ao seu desenvolvimento.

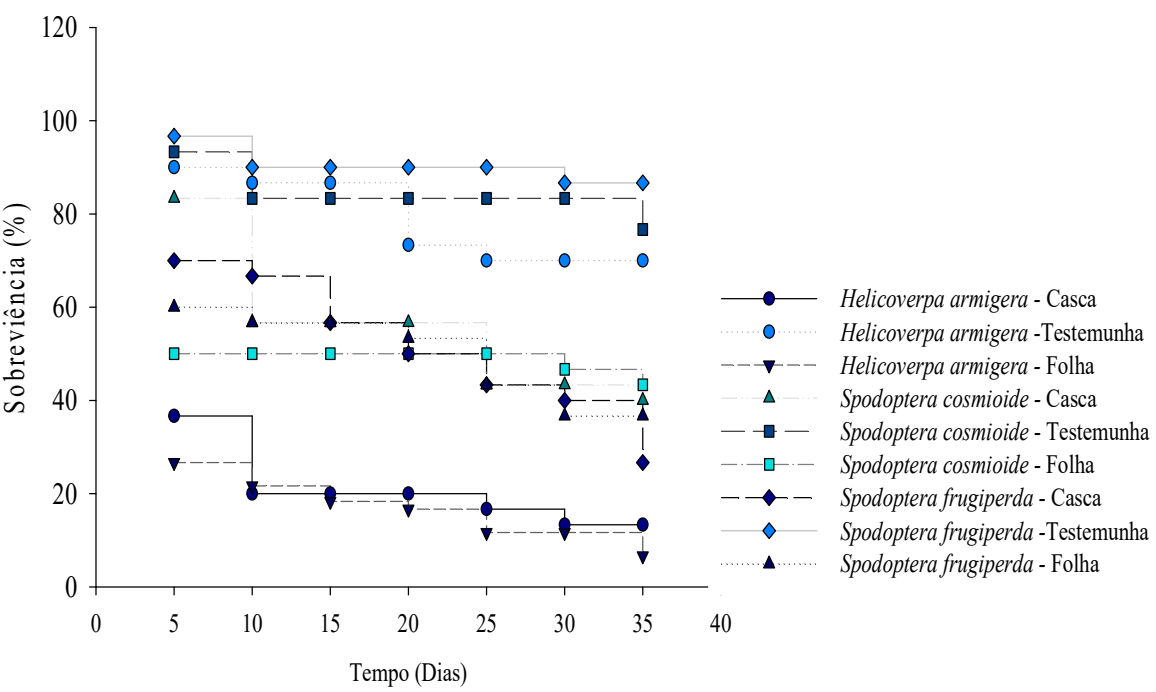

Figura 1. Análise de sobrevivência de Helicoverpa armigera, Spodoptera cosmioides e Spodoptera frugiperda, após a aplicação tópica do extrato etanólico de folhas e casca de Anadenanthera macrocarpa (Fabaceae: Mimosoideae) até 35 dias após o início do experimento, calculada pelo procedimento de curva de sobrevivência do estimador.

Figure 1. Survival analysis of Helicoverpa armigera, Spodoptera cosmioides and Spodoptera frugiperda, after the topical application of the ethanolic extract of leaves and bark of Anadenanthera macrocarpa (Fabaceae: Mimosoideae) up to 35 days after the start of the experiment, calculated by the curve survival of the estimator.

Tabela 3. Médias ( \pm DP) da duração da fase larval de Helicoverpa armigera, Spodoptera cosmioides e Spodoptera frugiperda, após a aplicação tópica do extrato etanólico das folhas e casca de Anadenanthera macrocarpa (Fabaceae: Mimosoideae).

Table 3. Mean ( \pm SD) of the larval phase duration of Helicoverpa armigera, Spodoptera cosmioides and Spodoptera frugiperda, after topical application of the leaves and bark extract of Anadenanthera macrocarpa (Fabaceae: Mimosoideae).

\begin{tabular}{ccc}
\hline Tratament & Longevidade Larval & Longevidade da pupa \\
\hline \multicolumn{3}{c}{ Helicoverpa armigera } \\
\hline casca & $25,2 \pm 1,01$ & $11,01 \pm 0,16$ \\
controle & $19,4 \pm 0,73$ & $10,31 \pm 019$ \\
folha & $26,025 \pm 0,53$ & $10,98 \pm 0,18$ \\
\hline $\mathrm{F}$ & $3,92^{\mathrm{NS}}$ & $1,29^{\mathrm{NS}}$ \\
$\mathrm{CV}(\%)$ & 15,49 & 6,39 \\
\hline \multicolumn{3}{c}{ Spodoptera cosmioides } \\
\hline casca & $33,45 \pm 0,62$ & $11,18 \pm 0,12$ \\
controle & $28,05 \pm 0,58$ & $10,81 \pm 0,10$ \\
folha & $29,95 \pm 1,19$ & $11,54 \pm 0,09$ \\
\hline $\mathrm{F}$ & $2,09^{\mathrm{NS}}$ & $2,31^{\mathrm{NS}}$ \\
$\mathrm{CV}(\%)$ & 12,41 & 4,31 \\
\hline \multicolumn{3}{c}{ Spodoptera frugiperda } \\
\hline casca & $35,20 \pm 0,15 \mathrm{a}$ & $11,72 \pm 0,25$ \\
controle & $24,95 \pm 0,40 \mathrm{~b}$ & $10,17 \pm 0,13$ \\
folha & $25,20 \pm 0,15 \mathrm{~b}$ & $10,35 \pm 0,24$ \\
\hline $\mathrm{F}$ & $99,49^{* *}$ & $3,12^{\mathrm{NS}}$ \\
CV $(\%)$ & 4,12 & 8,94 \\
\hline
\end{tabular}

Médias seguidas por mesma letra não diferem entre si pelo teste de Tukey a $5 \%$ de probabilidade.
Tabela 4. Médias ( $\pm \mathrm{DP})$ da massa das pupas de Spodoptera frugiperda, Spodoptera cosmioides e Helicoverpa armigera, após a aplicação tópica do extrato etanólico das folhas e casca de Anadenanthera macrocarpa (Fabaceae: Mimosoideae).

Table 4. Averages ( \pm SD) of the pupal mass of Spodoptera frugiperda, Spodoptera cosmioides and Helicoverpa armigera, after the topical application of the leaves and bark extract of Anadenanthera macrocarpa (Fabaceae: Mimosoideae).

\begin{tabular}{|c|c|c|}
\hline Tratamento & Massa larval $(\mathrm{g})$ & Massa das pupas (g) \\
\hline \multicolumn{3}{|c|}{ Helicoverpa armigera } \\
\hline casca & $0,41 \pm 0,00 \mathrm{a}$ & $0,18 \pm 0,00 \mathrm{a}$ \\
\hline controle & $0,42 \pm 0,03 \mathrm{a}$ & $0,16 \pm 0,01 \mathrm{~b}$ \\
\hline folha & $0,22 \pm 0,01 \mathrm{~b}$ & $0,13 \pm 0,01 \mathrm{c}$ \\
\hline $\mathrm{F}$ & $26,38^{* *}$ & $101,16^{* *}$ \\
\hline $\mathrm{CV}(\%)$ & 15,24 & 5,69 \\
\hline \multicolumn{3}{|c|}{ Spodoptera cosmioides } \\
\hline casca & $0,53 \pm 0,02 \mathrm{~b}$ & $0,37 \pm 0,00 \mathrm{~b}$ \\
\hline controle & $0,60 \pm 0,00 \mathrm{a}$ & $0,40 \pm 0,00 \mathrm{a}$ \\
\hline folha & $0,48 \pm 0,01 \mathrm{c}$ & $0,34 \pm 0,01 \mathrm{c}$ \\
\hline F & $90,42 * *$ & $9,15^{* *}$ \\
\hline CV $(\%)$ & 2,97 & 5,82 \\
\hline \multicolumn{3}{|c|}{ Spodoptera frugiperda } \\
\hline casca & $0,24 \pm 0,02 \mathrm{c}$ & $0,10 \pm 0,00 \mathrm{c}$ \\
\hline controle & $0,45 \pm 0,02 \mathrm{a}$ & $0,19 \pm 0,00 \mathrm{a}$ \\
\hline folha & $0,34 \pm 0,01 \mathrm{~b}$ & $0,12 \pm 0,00 \mathrm{~b}$ \\
\hline $\mathrm{F}$ & $8,95^{* *}$ & $109,67^{* *}$ \\
\hline $\mathrm{CV}(\%)$ & 24,17 & 8,02 \\
\hline
\end{tabular}

Médias com mesma letra não diferem entre si pelo teste de tukey a $5 \%$ de probabilidade. 
Embora as plantas apresentem boa atividade inseticida, os mecanismos de sua ação como inseticidas naturais ainda não são bem conhecidos. Esses mecanismos podem atuar em vários alvos em insetos e mamíferos, especialmente no sistema nervoso, incluindo canais de cloreto de ácido $\gamma$-aminobutírico (GABA), receptores de octopamina, receptores de tiramina, acetilcolinesterase, receptores nicotínicos de acetilcolina (nAChR), canais de sódio entre outros alvos (KRASOWSKI et al., 2002; HALL et al., 2004; GARCIA et al., 2006; ANDERSON; COATS, 2012). Portanto, o modo de ação depende do composto mais abundante da planta e pode interferir de várias formas no desenvolvimento e na biologia do inseto, em relação às espécies aqui estudadas houve redução de peso para $S$. frugiperda e interferência no estágio larval e pupal para ambas as espécies.

Experimentos realizados por Tanzubil e McCafferry (1990) com óleo de semente de Azadirachtina indica (Meliaceae) sobre Spodoptera exempta (Walker), observaram que a redução do crescimento aliada à mortalidade ocorre em razão do alto gasto energético empregado pelos insetos para quebra das substâncias tóxicas presentes em seu organismo ao invés de direcionar este custo energético para o desenvolvimento de larvas e pupas.

A redução no peso das pupas dos insetos submetidos aos extratos aquosos de folhas e casca de $A$. macrocarpa pode ser em função de alterações provocadas na fase larval. Segundo Lima et al. (2006) o peso das pupas está relacionado ao desempenho do inseto na fase larval, ou seja, o aumento ou redução do consumo de alimento pelas lagartas aumenta ou diminui também o peso das pupas. Uma vez que houve redução no consumo alimentar de lagartas submetidas aos extratos resultou em pupas com peso bastante reduzido em relação às lagartas submetidas ao tratamento controle.

As plantas produzem uma diversidade de compostos químicos que podem atuar na defesa contra patógenos ou insetos (PINTO-ZEVALLOS et al., 2013), isso ocorre devido a produção de substâncias não palatáveis, repelentes ou toxicas (KLIEBENSTEIN et al., 2001, KOORNNEEF et al., 2004), as quais representam um sistema de defesa química em que os produtos de hidrólise são liberados após a ruptura da estrutura celular durante o ataque de insetos (COLLINGE et al., 2010), no caso da $A$. macrocarpa esses compostos afetaram a sobrevivência e o desenvolvimento das espécies lepidópteros testadas.

Os principais componentes dos extratos do angico-preto são os flavonoides, taninos, terpenos e esteroides que estão associados a muitas atividades biológicas de organismos vivos (GUTIERREZ-LUGO et al., 2004; WEBER et al., 2011; SARTORI et al., 2014; SOUZA et al., 2013). Portanto, a presença ou sinergia dos compostos encontrados nos extratos etanólicos de folhas e casca de A. macrocarpa resultaram na mortalidade e efeitos sobre o comportamento e desenvolvimento das três espécies de lepidópteros testadas. Estudos realizados por Boiça Júnior et al. (2013) e Carvalho Neto et al. (2017), extratos botânicos e a influência dos compostos secundários sobre a biologia e os padrões de comportamento de alguns artrópodes-praga têm sido evidenciada, mostrando o efeito prejudicial dos compostos fenólicos, taninos, terpenos e saponinas em ovos, larvas, pupas e fases adultas de diferentes insetos e ácaros, nos quais os terpenos são notáveis em todos os estudos.

\section{CONCLUSÕES}

Os compostos químicos presentes nos extratos etanólicos, apresentam efeitos negativos nas diferentes fases biológicas de $S$. frugiperda, $S$. cosmoides e $H$. armigera. Os tratamentos de folhas e das cascas de A. macrocarpa foram eficientes na redução da sobevivência, da massa larval e de pupas em $S$. frugiperda e $S$. cosmioides, enquanto para $H$. armigera os extratos da folha apresentaram maior eficiência.

\section{REFERÊNCIAS}

ALMEIDA, M. L. S.; OLIVEIRA, A. S.; CARVALHO, G. S.; SILVA, L. S.; SILVA, L. B., SANTOS, E. C. B.; CASARIN, F. E. Toxicity of Croton urucurana Against the Subterranean Termite Heterotermes sulcatus (Isoptera: Rhinotermitidae). Journal of Entomology, London, v.13, p.48-54, 2016.2 DOI: http://dx.doi.org/10.3923/je.2016.48.54

ANDERSON, J. A.; COATS, J. R. Acetylcholinesterase inhibition by nootkatone and carvacrol in arthropods. Pesticide Biochemistry Physiology, v. 102, n. 2, p. 124128, 2012.2 DOI: https://dx.doi.org/10.1016/j.pestbp.2011.12.002

BOIÇA JÚNIOR, A. L.; JANINI, J. C.; SOUZA, B. H. S. de; RODRIGUES, N. E. L. Efeito de cultivares de repolho e doses de extrato aquoso de nim na alimentação e biologia de Plutella xylostella (Linnaeus) (Lepidoptera: Plutellidae). Bioscience Journal, Uberlandia, v. 29, p. 2231, 2013.

BUENO, R. C. O. F.; BUENO, A. F.; MOSCARDI, F.; PARRA, J. R. P.; HOFFMANN-CAMPO, C. B. Lepidopteran larva consumption of soybean foliage: basis for developing multiple-species economic thresholds for pest management decisions. Pest Management Science, Sussex, v. 67, n. 2, p. 170-174, 2011. DOI: https://dx.doi.org10.1002/ps.2047

CABEZAS, M. F.; NAVA, D. E.; GEISSLER, L. O.; MELO, M.; GARCIA, M. S.; KRÜGER, R. Development and leaf consumption by Spodoptera cosmioides (Walker) (Lepidoptera: Noctuidae) reared on leaves of Agroenergy Crops. Neotropical Entomology, Londrina, v. 42, n. 6, p. 588-594, 2013. DOI: https://dx.doi.org/10.1007/s13744013-0169-6

CARVALHINHO, D. T.; RODRIGUES, R. H. F.; CARNEIRO, E.; LOPES, G. N.; SILVA, L. B.; PAVAN, B. E. Toxicidade de extratos de Anadenanthera macrocarpa (Fabaceae: Mimosoideae) e Bougainvillea buttiana (Nyctaginaceae) para lepidópterospraga. Biotemas, Florianópolis, v. 30, p. 15-24, 2017. DOI: https://doi.org/10.5007/2175-7925.2017v30n2p15

CARVAlHO NETO, M. F.; GERVÁSIO, R. C. R. G.; ARAÚJO, E. C. C.; ALMEIDA, J. C.; GUIMARÃES, A. L. Phytochemical profile of cansanção nettle extracts and their bioactivities on cabbage caterpillar. Pesquisa agropecuária brasileira, Brasília, v. 52, n. 10, p. 841-848, 2017. DOI: http://dx.doi.org/10.1590/s0100204x2017001000003

CHAN, Y. S.; ZHANG, Y.; NG, T. B. Brown kidney bean Bowman-Birk trypsin inhibitor is heat and $\mathrm{pH}$ stable and exhibits antiproliferative activity. Applied Biochemistry and Biotechnology, Washington, v. 169, n. 4, p. 13061314, 2013. DOI: https://dx.doi.org/10.1007/s12010-0129998-8 
COLLINGE，D. B.; JORGENSEN, H. J.; LUND, O. S.; LYNGKJAER, M. F. Engineering pathogen resistance in crop plants: current trends and future prospects. Annual Review of Phytopathology, Palo Alto, v. 48, p. 269-291, 2010. DOI: https://dx.doi.org/10.1146/annurev-phyto073009-114430

CZEPAK, C.; ALBERNAZ, K. C.; VIVIAN, L. M.; GUIMARÃES, H. O.; CARVALHAIS, T. Primeiro Registro de Ocorrência de Helicoverpa armigera (Hübner) (Lepidoptera: Noctuidae) no Brasil. Pesquisa Agropecuária Tropical, Goiânia, v. 43, n. 1, p. 110-113, 2013. DOI: http://dx.doi.org/10.1590/S198340632013000100015

DEQUECH, S. T. B.; CAMERA, C.; STURZA, V. S.; RIBEIRO, L. P.; QUERINO, R. B.; PONCIO, S Population fluctuation of Spodoptera frugiperda eggs and natural parasitism by Trichogramma in maize. Acta Scientiarum Agronomy, Maringá, v. 35, n. 3, p.295-300, 2013.

DOI: https://dx.doi.org/10.4025/actasciagron.v35i3.16769

DINESH, D. S.; KUMARI, S.; KUMAR, V.; DAS, P. The potentiality of botanicals and their products as an alternative to chemical insecticides to sandflies (Diptera: Psychodidae): a review. Journal of Vector Borne Disease, v. 51, p. 1-7, 2014.

FINNEY D. J. Probit analysis: a statistical treatment of the sigmoid response curve. Cambridge: Cambridge University Press, 1947. 256 p.

FONSECA, W. L.; ALMEIDA, F. A.; LEITE, M. L. T.; RAMBO, A. P. P.; PETTER, F. A.; OLIVEIRA, A. M.; CARVALHO, R. M.; ALCÂNTARA NETO, F., PEREIRA, F. F. Bioactivity of aqueous extracts of Anadenanthera macrocarpa to Meloidogyne incognita in cotton crop. Australian Journal of Crop Science, v. 11, n. $2, \quad$ p. $156-161,2017 . \quad$ DOI: https://dx.doi.org/10.21475/ajcs.17.11.02.p203

FOUAD, H. A.; FARONI, L. R. D.; TAVARES, W. D.; RIBEIRO, R. C.; FREITAS, S. D.; ZANUNCIO, J. C. Botanical extracts of plants from the Brazilian Cerrado for the integrated management of Sitotroga cerealella (Lepidoptera: Gelechiidae) in stored grain. Journal of Stored Products Research, Oxford, v. 57, p. 6-11, 2014 DOI: https://doi.org/10.1016/j.jspr.2014.01.001

GARCIA, D. A.; BUJONS, J.; VALE, C.; SUNOL, C. Allosteric positive interaction of thymol with the GABAA receptor in primary cultures of mouse cortical neurons. Neuropharmacology, New York, v. 50, n. 1, p. 25-35, 2006.

DOI: https://doi.org/10.1016/j.neuropharm.2005.07.009

GUAZINA, R. A.; DEGRANDE, P. E.; SOUZA, E. P.; GAUER, E. Danos da lagarta Helicoverpa armigera (Hübner, 1805) (Lepidoptera: Noctuidae) em plântulas de soja. Revista de Ciências Agroveterinárias, Lages, v. 18, n. $1, \quad$ p. $41-46, \quad 2019$. DOI: http://dx.doi.org/10.5965/223811711812019041

GUTIERREZ-LUGO， M. T.; DESCHAMPS， J. D.; HOLMAN, T. R.; SUAREZ, E.; TIMMERMENN, B. N. Lipoxygenase inhibition by Anadanthoflavone, a new flavonoid from the aerials parts of Anadenanthera columbrine. Planta Medica, Stuttgart, v. 70, n. 3, p. 263265, 2004. DOI: https://dx.doi.org/10.1055/s-2004818920
HALL, A. C.; TURCOTTE, C. M.; BETTS, B. A.; YEUNG, W. Y.; AGYEMAN, A. S.; BURK, L. A. Modulation of human GABAA and glycine receptor currents by menthol and related monoterpenoids. European Journal of Pharmacology, Amsterdam, v. 506, n. 1, p. 9-16, 2004. DOI: https://dx.doi.org/10.1016/j.ejphar.2004.10.026

HARDSTONE, M. C.; SCOTT, J. G. Is Apis mellifera more sensitive to insecticides than other insects?. Pest Management Science, Sussex, v. 66, n. 11, p. 1171-1180, 2010.

ISMAN, M. B. Botanical insecticides, deterrents, and repellents in modern agriculture and an increasingly regulated world. Annual Review of Entomology, Stanford, v. 51, p. 45-66, 2006. DOI: https://dx.doi.org/10.1146/annurev.ento.51.110104.15114

KASTEN, J. R. P.; PRECETTI, A. A. C. M.; PARRA, J. R. P. Dados biológicos comparativos de Spodoptera frugiperda (J.E. SMITH, 1797) em duas dietas artificiais e substrato natural. Revista de Agricultura, v. 53, p. 69-78, 1978.

KLIEBENSTEIN, D. J.; KROYMANN, J.; BROWN, P.; FIGUTH, A.; PEDERSEN, D.; GERSHENZON, J.; MITCHELL-OLDS, T. Genetic Control of Natural Variation in Arabidopsis Glucosinolate Accumulation. Plant Physiology, Bethesda, v. 126, p. 811-825, 2001. DOI: https://dx.doi.org/10.1104/pp.126.2.811

KOORNNEEF, M.; ALONSO-BLANCO, $\quad$ C.; VREUGDENHIL, D. Naturally occurring genetic variation in Arabidopsis thaliana. Annual Review of Plant Biology, Palo Alto, v. 55, p. 141-172, 2004. DOI: https://dx.doi.org/10.1146/annurev.arplant.55.031903.141 605

KRASOWSKI, M. D.; HONG, X.; HOPFINGER, A. J.; HARRISON, N. L. 4D-QSAR analysis of a set of propofol analogues: mapping binding sites for an anesthetic phenol on the $\operatorname{GABA}(\mathrm{A})$ receptor. Journal of Medicinal Chemistry, Washington, v. 45, n. 15, p. 3210-3221, 2002. DOI: https://dx.doi.org/10.1021/jm010461a

LIMA, F. W. N.; OHASHI, O. S.; SOUZA, F. R. S.; GOMES, F. S. Avaliação de acessos de milho para resistência a Spodoptera frugiperda (Smith) (Lepidoptera: Noctuidae) em laboratório. Acta Amazônica, Manaus, v. 36, n. 2, p. 147-150, 2006. DOI: http://dx.doi.org/10.1590/S004459672006000200003

MARTINEZ, S. S.; EMDEN, H. F. Growth disruption, abnormalities and mortality of Spodoptera littoralis (Boisduval) (Lepidoptera: Noctuidae) caused by azadirachtin. Neotropical Entomology, Londrina, v.30, n.1, p.113-125, 2001. DOI: http://dx.doi.org/10.1590/S1519-566X2001000100017

MATOS, A. P.; NEBO, L.; CALEGARI, E. R.; BATISTAPEREIRA, L. G.; VIEIRA, P. C.; FERNANDES, J. B.; SILVA, M. F. G. F.; FERREIRA FILHO, P.; RODRIGUES, R.R. Atividade Biológica de Extratos Orgânicos de Trichilia spp. (Meliaceae) sobre Spodoptera frugiperda (J. E. Smith) (Lepidoptera: Noctuidae) em Dieta Artificial. BioAssay, Piracicaba, v. 1, p.1-7, 2006. DOI: https://dx.doi.org/10.14295/BA.v1.0.39

MELO, B. A.; MOLINA-RUGAMA, A.J.; HADDI, K.; LEITE, D.T.; OLIVEIRA, E. E. Repellency and Bioactivity of Caatinga Biome Plant Powders against Callosobruchus maculatus (Coleoptera: Chrysomelidae: 
Bruchinae). Florida Entomologist, Gainesville, v. 98, p. 417-423, 2015.

DOI: https://dx.doi.org/10.1653/024.098.0204

PINTO-ZEVALLOS, D. M., MARTINS, C. B. C.; PELlEGRINO, A. C.; ZARBIN, P. H. G. Compostos orgânicos voláteis na defesa induzida das plantas contra insetos herbívoros. Química Nova, São Paulo, v. 36, n. 9, p. 1395-1405, 2013, DOI: http://dx.doi.org/10.1590/S0100-40422013000900021

REGNAULT-ROGER, C.; VINCENT, C.; ARNASON, J. T. Essential oils in insect control: low-risk products in a highstakes world. Annual Review of Entomology, Stanford, v. 57, p. $405-424, \quad 2012$. DOI: https://dx.doi.org/10.1146/annurev-ento-120710-100554

SANTOS, R. F. E. P.; CONSERVA, L. M.; BASTOS, M. L. A.; CAMPESATTO, E. A. Avaliação do potencial biológico da Tabebuia aurea (Silva Manso) como fonte de moléculas bioativas para atividade antimicrobiana, antiedematogênica e antirradicalar. Revista Brasileira de Plantas Medicinais, Botucatu, v. 17, n. 4, p. 1159-1168, 2015. DOI: http://dx.doi.org/10.1590/1983-084x/14_168

SANTOS, W. L.; FREIRE, M. G. M.; BOGORNI, P. C.; VENDRAMIM, J. D.; MACEDO, M. L. R. Effect of the aqueous extracts of the seeds of Talisia esculenta and Sapindus saponaria on fall armyworm. Brazilian Archives of Biology and Technology, Curitiba, v. $51, \quad$ n. 2, p. $373-383,2008$. DOI: http://dx.doi.org/10.1590/S1516-89132008000200018

SARTORI, C. J.; CASTRO, A. H. F.; MORI, F. A. Teores de Fenóis Totais e Taninos nas Casca de Angico-vermelho (Anadenanthera peregrina). Floresta e Ambiente, Seropédica, v. 21, n. 3, p. 394-400, 2014. DOI: http://dx.doi.org/10.1590/2179-8087.061113

SAS INSTITUTE. SAS user's manual, version 9.1. 2002. Disponível em: https://support.sas.com/documentation/onlinedoc/91pdf/s asdoc_91/stat_ug_7313.pdf >.

SILVA, W.; FREIRE, M. das. G. M.; PARRA, J. R. P.; S. MARANGONI; MACEDO, M. L. R. Evaluation of the Adenanthera pavonina seed proteinase inhibitor (ApTI) as a bioinsecticidal tool with potential for the control of Diatraea saccharalis. Process Biochemistry, Amsterdam, v. 47 , n. 2, p. 257-263, 2012. DOI: https://dx.doi.org/10.1016/j.procbio.2011.11.002

SOUZA, J. N. P.; CANDOTTI, J. G.; AMPARO, T. R.; COELHO, F. F.; RODRIGUES, I. V.; DOS SANTOS, O. D. H.; DE MEDEIROS, L. F. T.; FURTADO, N. A. J. C.; DE SOUSA, H. C.; DE SOUZA, G. H. B. Bioprospecção das atividades antioxidante e antimicrobiana de espécies vegetais medicinais coletadas em Ouro Preto-MG. Revista Eletrônica de Farmácia, Goiânia, v. 10, n. 1, p. 1-15, 2013. DOI: http://dx.doi.org/10.5216/ref.v10i1.18635

SPECHT, A.; SOSA-GOMEZ, D. R.; PAULA-MORAES, S. V.; YANO, S. A. C. Identificação morfológica e molecular de Helicoverpa armigera (Lepidoptera: Noctuidae) e ampliação de seu registro de ocorrência no Brasil. Pesquisa Agropecuária Brasileira, Brasília, v. 48, n. 6, p. 689-692, 2013.

TANZUBIL, P. B.; MCCAFERRY, A. R. Effects of azadirachtin and aqueous neem seed extracts on survival, growth and development of the African armyworm, Spodoptera exempta. Crop Protection, Guilford, v. 9, n.
5, p.383-386, 1990. DOI: https://dx.doi.org/10.1016/02612194(90)90012-V

WAR, A. R.; SHARMA, H. C.; PAULRAJ, M. G.; WAR, M. Y.; IGNACIMUTHU, S. Herbivore induced plant volatiles Their role in plant defense for pest management. Plant Signaling \& Behavior, v. 6, p. 1973-1978, 2011. DOI: https://dx.doi.org/10.4161/psb.6.12.18053

WEBER, C. R.; SOARES, C. M. L.; LOPES, A. B. D.; SILVA, T. S.; NASCIMENTO, M. S.; XIMENES, E. C. P. A. Anadenanthera colubrina: um estudo do potencial terapêutico. Revista Brasileira de Farmácia, Rio de Janeiro, v. 92, n. 4, p. 235-244, 2011. 